\title{
Sobre as restrições impostas ao comportamento estatístico de interfências externas em enlaces via satélite
}

\author{
Antonio José S. Baptista e José Mauro P. Fortes
}

\begin{abstract}
Resumo-Este artigo apresenta uma técnica para a determinação de máscaras que limitam o comportamento estatístico do agregado de interferências externas que afetam um determinado enlace via satélite, visando garantir o desempenho adequado do enlace. A técnica proposta utiliza uma representação em base de funções ortonormais para a densidade de probabilidade da degradação devido a interferências externas e a solução de um problema de otimização com restrições convenientemente definido. Um exemplo de aplicação da técnica proposta a uma situação de interesse real é também apresentada.
\end{abstract}

Palavras-Chave-interferência, máscaras limitantes, degradação por chuvas, otimização.

Abstract-This paper presents a technique to determine masks that limit the statistical behavior of the aggregate external interference affecting a satellite link so that an adequate link performance is guaranteed. The proposed technique uses a series representation, in a basis of orthonormal functions, for the probability density function of the degradation due to external interferences and the solution of a conveniently defined constrained optimization problem. An application example involving a real situation is also presented.

Keywords-interference, limiting masks, degradation due to rain, optimization.

\section{INTRODUÇÃo}

Quando diversos sistemas de comunicações compartilham uma determinada faixa de frequiências, cada um dos sistemas envolvidos opera sujeito às interferências geradas pelos demais. Nesta situação é importante a realização de estudos e análises que visam definir as limitações que devem ser impostas aos sistemas interferentes de modo a garantir a proteção do sistema vítima. Estas análises são usualmente complexas e dependem fortemente das características técnicas dos sistemas envolvidos. O procedimento usual para este tipo de estudo inclui as seguintes etapas: (i) definição, por parte dos operadores do sistema vítima, dos requisitos de desempenho pretendido para seu sistema; (ii) definição, por parte dos operadores do sistema interferente, do tipo de limitação a ser imposta aos sinais transmitidos por seus satélites e/ou suas estações terrenas (e.g., máscaras para o comportamento estatístico da potência interferente, máscaras para o comportamento estatístico da densidade de fluxo de potência interferente); (iii) obtenção de uma máscara que, ao limitar o comportamento estatístico

Antonio José S. Baptista, Centro de Projeto de Navios - Marinha do Brasil, baptista_mail@yahoo.com.br; José Mauro P. Fortes, Centro de Estudos em Telecomunicações da PUC-Rio,jmfortes@cetuc.puc-rio.br. Este trabalho foi parcialmente financiado pelo Conselho Nacional de Desenvolvimento Científico e Tecnológico - CNPq. da potência das transmissões do sistema interferente, garanta, sem impor restrições desnecessárias ao sistema interferente, que os requisitos de desempenho do sistema sejam satisfeitos. Dada a complexidade da última etapa deste processo, a definição da máscara adequada é usualmente feita de maneira iterativa: partindo-se de uma determinada máscara, verifica-se os requisitos de desempenho especificados pelo sistema vítima é atendido. Caso eles não sejam atendidos, a máscara inicial é modificada de modo aumentar a restrição às transmissões do sistema interferente. Caso ele seja atendido com folga, a mascara inicial é modificada de modo a diminuir as restrições impostas aos sinais transmitidos. Este procedimento é feito iterativamente, conduzindo à definição de uma máscara que garanta a proteção do sistema vítima, sem impor restrições indevidas às potências de transmissão do sistema interferente.

Técnicas para a obtenção de máscaras que garantem a proteção de enlaces de sistemas do Serviço Fixo por Satélite (geoestacionários e não geoestacionários) limitando o comportamento estatístico da potência interferente, que não utilizam a iteratividade descrita acima, podem ser encontradas no Anexo I da Recomendação S.1323 da União Internacional de Telecomunicações [1]. As metodologias apresentadas neste anexo consideram o efeito conjunto das degradações provocadas por chuvas e por interferências externas. Entretanto, devido à complexidade destas metodologias e à dificuldade envolvida na sua aplicação a sistemas reais, os exemplos de aplicação apresentados em [1] são de caráter acadêmico e envolvem uma modelagem muito simples para o comportamento aleatório da interferência, que não reflete situações de real interesse. Este fato motivou o presente trabalho, no qual, através da representação da densidade de probabilidade da degradação devido a interferências externas em base de funções ortonormais e da solução de um problema de otimização convenientemente definido, foi possível desenvolver uma técnica que permite aplicar, de maneira sistemática, metodologias como as descritas em [1] a situações de real interesse que envolvem os modelos de propagação usualmente utilizados na estimação da atenuação por chuvas e uma caracterização genérica para comportamento estatístico da interferência.

Na Seção II descreve-se, e maneira geral, a modelagem matemática que suporta as metodologias de obtenção de máscaras para limitar o comportamento estatístico da potência interferente e que, como as descritas no Anexo I de [1], consideram o efeito conjunto da atenuação por chuvas e de interferências externas. Na Seção III, esta modelagem é complementada com o estabelecimento de uma representação 
em série de funções para a densidade de probabilidade da degradação devido a interferências e com a definição um problema de otimização convenientemente escolhido, cuja solução produz uma máscara adequada à limitação do comportamento estatístico da interferência. Finalmente, na Seção IV, é apresentado um exemplo que ilustra a aplicação da técnica proposta a uma situação real envolvendo um sistema vítima que utiliza satélite geoestacionário. Conclusões são apresentadas na Seção V.

\section{Modelagem Matemática}

Para garantir o desempenho adequado de um enlace via satélite é usual estabelecer um conjunto de restrições que devem ser satisfeitas pela taxa de erro de bit (BER) do enlace. Isto é feito limitando-se as percentagens de tempo durante as quais níveis pré-estabelecidos de BER podem ser excedidos. De certa forma, estas restrições podem ser expressas como

$$
P\left(b>B E R_{j}\right) \leq p_{j} \quad ; j=1, \ldots, m
$$

onde $b$ é uma variável aleatória que caracteriza a taxa de erro de bit do enlace, $\left\{B E R_{j}, j=1, \ldots, m\right\}$ denotam níveis préespecificados de $\operatorname{BER}$ e $\left\{p_{j}, j=1, \ldots, m\right\}$ são valores de probabilidade que refletem a percentagem de tempo durante as quais estes valores podem ser excedidos. Note que a taxa de erro de bit está sendo modelada por uma variável aleatória devido à sua dependência de fatores de degradação como, por exemplo, a atenuação por efeito de chuvas e a presença de interferências externas. Esta dependência pode ser estabelecida conforme descrito a seguir. Em primeiro lugar, seja $e$ a variável aleatória que caracteriza a razão $E_{b} / N_{0}$ (razão entre a energia por bit e o nível espectral de ruído térmico, expressa em $\mathrm{dB}$ ) e $f(e)$ a função que representa sua influência sobre a BER, ou seja,

$$
b=f(e)
$$

A função $f$ é decrescente e, conseqüentemente,

$$
P\left(b>B E R_{j}\right)=P\left(e<\left(E_{b} / N_{0}\right)_{j}\right) \leq p_{j} \quad ; j=1, \ldots, m
$$

com os valores $B E R_{j}$ e $\left(E_{b} / N_{0}\right)_{j}$ relacionados através da função $f$, ou seja,

$$
B E R_{j}=f\left(\left(E_{b} / N_{0}\right)_{j}\right) \quad ; j=1, \ldots, m
$$

Note que (3) permite estabelecer uma equvalência entre o conjunto de pares $\left\{\left(B E R_{j}, p_{j}\right) ; j=1, \ldots, m\right\}$ e o conjunto de pares $\left\{\left(\left(E_{b} / N_{0}\right)_{j}, p_{j}\right) ; j=1, \ldots, m\right\}$. Deste modo, as restrições de desempenho em (1) são equivalentes às restrições

$$
P\left(e<\left(E_{b} / N_{0}\right)_{j}\right) \leq p_{j} \quad ; j=1, \ldots, m
$$

Observe que a variável aleatória $e \operatorname{em}(5)$ corresponde à razão $E_{b} / N_{0}$ degradada, significando que ela é igual à diferença entre a razão $E_{b} / N_{0}$ correspondente à situação de céu claro e a degradação $z$ devido a chuvas e/ou interferências externas, ambas expressas em dB. Assim,

$$
e=\left(E_{b} / N_{0}\right)_{C S}-z
$$

onde $\left(E_{b} / N_{0}\right)_{C S}$ denota a razão $E_{b} / N_{0}$ de céu claro.
Sejam $x$ e $y$ variáveis aleatórias caracterizando, respectivamente, as degradações de $E_{b} / N_{0}$, em $\mathrm{dB}$, devido a chuvas e a interferências externas. Deste modo, quando a degradação devido a chuvas é a única a ser considerada, a variável aleatória $z$ em (6) é igual a $x$. No caso em que ambas as degradações (devido a chuvas e devido a interferências externas) são consideradas, $z=x+y$. Estas duas situações são consideradas nas seções seguintes.

\section{A. Degradação devido a chuvas apenas}

O Recommends 3 da Recomendação ITU-R S.1323 [1] estabelece que, para uma rede do Serviço Fixo por Satélite que utiliza satélite em órbita geoestacioária, as interferências externas causadas por estações terrenas ou espaciais de outras redes por satélite operando na mesma faixa de freqüências devem ser responsáveis por, no máximo, um décimo da percentagem de tempo durante a qual um determinado nível de BER (ou $C / N$, ou $E_{b} / N_{0}$ ) pode ser excedido, sendo esta percentagem de tempo especificada nos objetivos de desempenho de curto prazo da rede vítima. Considera-se então que a degradação devido a chuvas pode ser responsável por, no máximo, nove décimos da percentagem de tempo durante a qual um determinado nível de BER pode ser excedido, mesmo quando não existem interferências externas presentes. Isto significa que, neste caso,

$$
P\left(e<\left(E_{b} / N_{0}\right)_{j}\right) \leq 0.9 p_{j} \quad ; j=1, \ldots, m
$$

Considerando- se que a degradação $z$ da razão $E_{b} / N_{0}$ em (6) é igual à degradação $x$ devido a chuvas apenas, tem-se

$$
e=\left(E_{b} / N_{0}\right)_{C S}-x
$$

e, conseqüentemente,

$$
P\left(e<\left(E_{b} / N_{0}\right)_{j}\right)=P\left(x>X_{j}\right) ; j=1, \ldots, m
$$

com os valores $\left\{X_{j}, j=1, \ldots, m\right\}$ dados por

$$
X_{j}=\left(E_{b} / N_{0}\right)_{C S}-\left(E_{b} / N_{0}\right)_{j}
$$

As restrições em (7) são então equivalentes a

$$
P\left(x>X_{j}\right) \leq 0,9 p_{j} \quad ; j=1, \ldots, m
$$

ou ainda,

$$
1-F_{x}\left(X_{j}\right) \leq 0,9 p_{j} \quad ; j=1, \ldots, m
$$

onde os níveis de atenuação por chuvas $\left\{X_{j}, j=1, \ldots, m\right\}$ são dados por (10) e $F_{x}(X)$ denota a função distribuição de probabilidade da variável aleatória $x$, definida por

$$
F_{x}(X)=P(x \leq X)
$$

A Figura 1 ilustra uma típica função $1-F_{x}(X)$ (usualmente denominada distribuição cumulativa de probabilidade) correspondente a atenuação devido a chuvas. Note que de acordo com as restrições em (12), esta distribuição cumulativa de probabilidade deve passar abaixo dos triângulos indicados na Figura 1. 


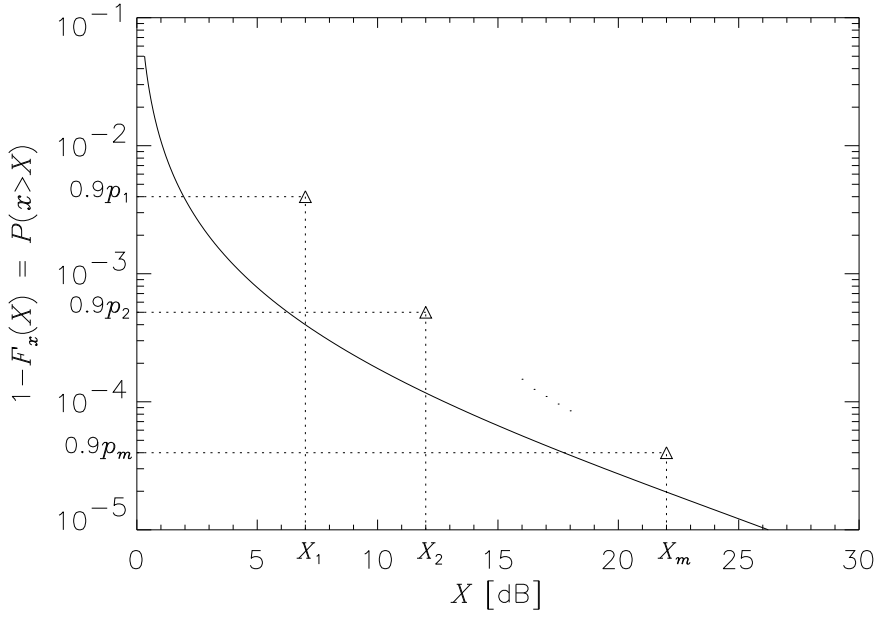

Fig. 1. Atenuação devido a chuvas: distribuição cumulativa de probabilidade típica.

\section{B. Degradação devido a chuvas e a interferências externas}

Neste caso, a variável aleatória $z$ em (6) pode ser escrita como

$$
z=x+y
$$

onde $x$ e $y$ são variáveis aleatórias caracterizando, respectivamente, as degradações de $E_{b} / N_{0}$, em $\mathrm{dB}$, devido a chuvas e a interferências externas. Considerando-se que estas duas variáveis aleatórias são estatisticamente independentes, a função distribuição de probabilidade de $z$ é dada por

$$
F_{z}(Z)=F_{x}(Z) * p_{y}(Z)
$$

o que significa que a função distribuição de probabilidade da degradação total $z$ é a convolução da função distribuição de probabilidade da degradação $x$ devido a chuvas com a função densidade de probabilidade da degradação $y$ devido a interferências externas. Note que, considerando-se (6), as restrições em (5) são equivalentes a

$$
P\left(z>Z_{j}\right) \leq p_{j} \quad ; \quad j=1, \ldots, m
$$

ou

$$
F_{z}\left(Z_{j}\right) \geq 1-p_{j} \quad ; \quad j=1, \ldots, m
$$

com os valores $\left\{Z_{j}, j=1, \ldots, m\right\}$ dados por

$$
Z_{j}=\left(E_{b} / N_{0}\right)_{C S}-\left(E_{b} / N_{0}\right)_{j}
$$

Em resumo, o problema a ser resolvido consiste em, dada a Função Distribuição de Probabilidade $F_{x}(X)$ da dagradação devido a chuvas, determinar, considerando o relacionamento em (15), uma Função Densidade de Probabilidade $p_{y}(Y)$ e, conseqüentemente, uma Função Distribuição de Probabilidade $F_{y}(Y)$ para degradação devido a interferências externas que garanta que às restrições em (18) sejam satisfeitas. Note que este problema possui inúmeras soluções. As metodologias descritas no Anexo I de [1] sugerem utilizar a distribuição cumulativa de probabilidade $C_{y}(Y)=1-F_{y}(Y)$ associada a qualquer uma destas soluções como uma máscara para o comportamento estatítico da degradação devido a interferências externas uma vez que os requisitos de desempenho em (1) são satisfeitos para qualquer degradação cujo comportamento estatístico corresponda a uma distribuição cumulativa de probabilidade que esteja abaixo de $C_{y}(Y)$. Dependendo da solução escolhida a máscara obtida pode ser mais, ou menos, restritiva.

Pode ser facilmente verificado [2] que o relacionamento entre a razão interfência-ruído térmico $i / n$ (razão entre a potência do sinal interferente e a potência de ruído térmico) e a degradação $y$ devido a interferências, quando ambas estão expressas em $\mathrm{dB}$, é dado por

$$
\frac{i}{n}=10 \log \left(10^{\frac{y}{10}}-1\right)
$$

Assim, a função distribuição de probabilidade da razão $i / n$ pode ser obtida a partir da função distribuição dew probabilidade da degradação $y$ através da relação

$$
F_{\frac{i}{n}}(\Gamma)=F_{y}\left(10 \log \left(10^{\frac{\Gamma}{10}}+1\right)\right)
$$

Esta relação permite obter uma máscara para o comportamento estatístico da razão interferência-ruído térmico, dada por $C_{\frac{i}{N}}(\Gamma)=1-F_{\frac{i}{N}}(\Gamma)$.

\section{Um PRoblema de OtimizaçÃo Conveniente}

Nesta seção, é definido um problema de otimização com restrições, cuja solução permite se chegar, de maneira sistemática, a uma das soluções possíveis para o problema estabelecido na Seção II. Além disso, para possibilitar a aplicação da técnica proposta a situações de interesse real, é proposta uma representação paramétrica para a função densidade de probabilidade da degradação $y$ devido a interferências, sendo consideradas as seguintes hipóteses: (i) a degradação $y$ é limitada ao intervalo $\left[Y_{\min }, Y_{\max }\right]$; (ii) permite-se a existência de uma probabilidade diferente de zero para as degradações iguais a $Y_{\min }$ e $Y_{\max }$ e (iii) no intervalo aberto $\left(Y_{\min }, Y_{\max }\right)$, a função densidade de probabilidade da degradação devido a interferências $p_{y}(Y)$ é contínua e diferenciável, e será representada por uma expansão em série de uma base de funções ortonormais contínuas e diferenciáveis no intervalo $\left(Y_{\min }, Y_{\max }\right)$. Sob estas hipóteses, a função densidade de probabilidade $p_{y}(Y)$ pode ser escrita como

$p_{y}(Y)=\alpha_{0} \delta\left(Y-Y_{\min }\right)+\alpha_{n+1} \delta\left(Y-Y_{\max }\right)+\sum_{i=1}^{n} \alpha_{i} \phi_{i}(Y)$

onde $\delta($.$) é a função impulso, \left\{\phi_{i}(Y), i=1, \ldots, n\right\}$ é o conjunto de funções ortonormais contínuas e diferenciáveis no intervalo $\left(Y_{\min }, Y_{\max }\right)$ e $\left\{\alpha_{0}, \ldots, \alpha_{n+1}\right\}$ o conjunto de parâmetros utilizados para representar a função $p_{y}(Y)$.

Note que, como

$$
\int_{Y_{\min }}^{Y_{\max }} p_{y}(Y) d Y=1
$$

é possível expressar o valor do parâmetro $\alpha_{n+1}$ em (21) como função dos demais parâmetros $\left(\left\{\alpha_{0}, \alpha_{1}, \ldots, \alpha_{n}\right\}\right)$. Temse assim,

$$
\alpha_{n+1}=1-\alpha_{0}-\sum_{i=1}^{n} \alpha_{i} c_{i}
$$


onde

$$
c_{i}=\int_{Y_{\min }}^{Y_{\max }} \phi_{i}(Y) d Y ; i=1,2, \ldots, n
$$

Conseqüentemente, $p_{y}(Y)$ pode ser escrita como função dos $n+1$ primeiros parâmetros $\left\{\alpha_{0}, \ldots, \alpha_{n}\right\}$, obtendo-se de (21),

$$
\begin{aligned}
p_{y}(Y)=\delta & \left(Y-Y_{\max }\right)+\alpha_{0}\left[\delta\left(Y-Y_{\min }\right)-\delta\left(Y-Y_{\max }\right)\right] \\
& +\sum_{i=1}^{n} \alpha_{i}\left[\phi_{i}(Y)-c_{i} \delta\left(Y-Y_{\max }\right)\right]
\end{aligned}
$$

com os coeficientes $c_{i}$ dados por (24). Substituindo-se (25) em (15) e considerando-se $Z=Z_{j}$, obtém-se

$$
\begin{aligned}
F_{z}\left(Z_{j}\right)=( & \left.1-\alpha_{0}\right) F_{x}\left(Z_{j}-Y_{\max }\right)+\alpha_{0} F_{x}\left(Z_{j}-Y_{\min }\right) \\
& +\sum_{i=1}^{n} \alpha_{i}\left[M_{i}\left(Z_{j}\right)-c_{i} F_{x}\left(Z_{j}-Y_{\max }\right)\right]
\end{aligned}
$$

onde

$$
M_{i}\left(Z_{j}\right)=\left.\phi_{i}(Z) * F_{x}(Z)\right|_{Z=Z_{j}} \quad ; \quad \begin{aligned}
& j=1,2, \ldots, m \\
& i=1,2, \ldots, n
\end{aligned}
$$

ou, em notação mais compacta,

$$
F_{z}\left(Z_{j}\right)=f_{j}^{\max }+\mathbf{k}_{j}^{T} \boldsymbol{\alpha} ; j=1,2, \ldots, m
$$

onde

$$
\begin{aligned}
f_{j}^{\max } & =F_{z}\left(Z_{j}-Y_{\max }\right) \\
\boldsymbol{\alpha} & =\left(\begin{array}{llll}
\alpha_{0} & \alpha_{1} & \cdots & \alpha_{n}
\end{array}\right)^{T}
\end{aligned}
$$

e

$$
\mathbf{k}_{j}=\mathbf{m}_{j}+f_{x}^{\max } \mathbf{c}
$$

Em (31),

$$
\mathbf{c}=\left(\begin{array}{llll}
1 & c_{1} & \cdots & c_{n}
\end{array}\right)^{T}
$$

$\operatorname{com}\left\{c_{i} ; i=1, \ldots, n\right\}$ dado por (24) e

$$
\mathbf{m}_{j}=\left(\begin{array}{llll}
f_{j}^{\min } & M_{1}\left(Z_{j}\right) & \cdots & \left.M_{n}(Z) j\right)
\end{array}\right)^{T}
$$

$\operatorname{com}\left\{M_{i}\left(Z_{j}\right) ; i=1,2, \ldots, n\right\}$ dado por (27) e

$$
f_{j}^{\text {min }}=F_{z}\left(Z_{j}-Y_{\min }\right)
$$

Observe que, como $F_{z}(Z)$ é uma função distribuição de probabilidade, é importante garantir que

$$
0 \leq F_{z}\left(Z_{j}\right) \leq 1 ; j=1,2, \ldots, m
$$

Por outro lado, as restrições em (17) impõem a condição

$$
F_{z}\left(Z_{j}\right) \geq 1-p_{j} \quad ; j=1,2, \ldots, m
$$

A partir de (35) e (36), verifica-se que os valores $\left\{F_{z}\left(Z_{j}\right) ; j=\right.$ $1,2, \ldots, m\}$ devem satisfazer às desigualdades

$$
1-p_{j} \leq F_{z}\left(Z_{j}\right) \leq 1 ; j=1,2, \ldots, m
$$

ou ainda, considerando (28),

$$
1-p_{j} \leq f_{j}^{\max }+\mathbf{k}_{j}^{T} \boldsymbol{\alpha} \leq 1 ; j=1,2, \ldots, m
$$

ou, de outra forma,

$$
1-p_{j}-f_{j}^{\max } \leq \mathbf{k}_{j}^{T} \boldsymbol{\alpha} \leq 1-f_{j}^{\max } ; j=1,2, \ldots, m
$$

que caracteriza um conjunto inicial de $2 m$ restrições para os parâmetros $\alpha_{0}, \alpha_{1}, \ldots, \alpha_{n}$ da função $p_{y}(Y)$ definida em (21).

Para que $p_{y}(Y)$ tenha as características próprias de uma função densidade de probabilidade, restrições adicionais têm que ser impostas aos parâmetros $\alpha_{0}, \alpha_{1}, \ldots, \alpha_{n}$. Obtem-se, então, a partir de (21), as restrições

$$
\begin{aligned}
& 0 \leq \alpha_{0} \leq 1 \\
& 0 \leq \alpha_{n+1} \leq 1
\end{aligned}
$$

considerando-se (23), as restrições em (41) se escrevem

$$
0 \leq 1-\alpha_{0}-\sum_{i=1}^{n} \alpha_{i} c_{i} \leq 1
$$

ou, em notação matricial,

$$
0 \leq \mathbf{c}^{T} \boldsymbol{\alpha} \leq 1
$$

com $\boldsymbol{\alpha}$ e c definidos, respectivamente, em (30) e (32).

Além disso, é necessário garantir que $p_{y}(Y) \geq 0$ para $Y \in$ $\left(Y_{\min }, Y_{\max }\right)$. Tem-se então, a partir de $(21)$,

$$
\sum_{i=1}^{n} \alpha_{i} \phi_{i}(Y) \geq 0 ; \quad \begin{aligned}
& Y \in\left(Y_{\min }, Y_{\max }\right) \\
& i=1,2, \ldots, n
\end{aligned}
$$

o que, em notação matricial, se escreve

$$
(\boldsymbol{\Phi}(Y))^{T} \boldsymbol{\alpha} \geq 0 ; \quad Y \in\left(Y_{\min }, Y_{\max }\right)
$$

onde $\boldsymbol{\Phi}(Y)$ é o vetor de dimensão $n+1$ definido por

$$
\boldsymbol{\Phi}(Y)=\left(\begin{array}{lll}
0 & \phi_{1}(Y) \cdots \phi_{n}(Y)
\end{array}\right)^{T}
$$

Assim, (40), (43) e (45) constituem restrições adicionais a serem satisfeitas pelos parâmetros $\alpha_{0}, \alpha_{1}, \ldots, \alpha_{n}$.

Para facilitar a implementação da restrição em (45), o intervalo $\left[Y_{\operatorname{mim}}, Y_{\max }\right]$ pode ser discretizado, considerando-se $N_{p}$ pontos igualmente espaçados, dados por

$$
Y_{k}=Y_{\min }+k \frac{Y_{\max }-Y_{\min }}{N_{p}-1} ; k=0,1, \ldots, N_{p}-1
$$

A restrição (45) é então substituída por

$$
\boldsymbol{\Phi}_{\mathbf{k}}{ }^{T} \boldsymbol{\alpha} \geq 0 \quad ; \quad k=0,1, \ldots, N_{p}-1
$$

onde $\boldsymbol{\Phi}_{\mathbf{k}}=\boldsymbol{\Phi}\left(Y_{k}\right)$ com $\boldsymbol{\Phi}(Y)$ dado por (46).

Neste ponto, observa-se que qualquer conjunto de parâmetros $\left\{\alpha_{0}, \alpha_{1}, \ldots, \alpha_{n}\right\}$ que satisfaça às restições em (39), (40), (43) e (45) ou (48) corresponde a uma densidade de probabilidade $p_{y}(Y)$ (ou seja, a um comportamento estatístico para a degradação devido a interferências externas) que garante que os requisitos de desempenho em (1) sejam atendidos. Suponha então que, dentre todos estes conjuntos, deseja-se determinar aquele que corresponde a uma função densidade de probabilidade $p_{y}(Y)$ para a qual a probabilidade de $y$ tomar valores num dado subconjunto $\mathcal{S}$ do intervalo $\left(Y_{\min }, Y_{\max }\right)$ seja máxima. Note que esta probabilidade se escreve

$$
P(y \in \mathcal{S})=\int_{\mathcal{S}} p_{y}(Y) d Y
$$

o que, considerando (21), se escreve

$$
P(y \in \mathcal{S})=\sum_{i=1}^{n} \alpha_{i} \int_{\mathcal{S}} \phi_{i}(Y) d Y
$$


ou ainda, em notação mais compacta,

$$
P(y \in \mathcal{S})=\mathbf{d}^{T} \boldsymbol{\alpha}
$$

onde $\boldsymbol{\alpha}$ é dado por (30) e $\mathbf{d}=\left(\begin{array}{lllll}0 & d_{1} & d_{2} & \ldots & d_{n}\end{array}\right)^{T}$ com

$$
d_{i}=\int_{\mathcal{S}} \phi_{i}(Y) d Y ; i=1, \ldots, n
$$

Fica assim fica definido um problema de otimização com restrições no qual deseja-se determinar o valor do vetor $\boldsymbol{\alpha}$ que maximiza a função objetivo dada por (51), sujeita às restrições (39), (40), (43) e (48).

Em resumo, deseja-se maximizar a função

$$
f(\boldsymbol{\alpha})=\mathbf{d}^{T} \boldsymbol{\alpha}
$$

sujeita às restrições

$$
\begin{aligned}
\mathbf{k}_{j}^{T} \boldsymbol{\alpha} & \geq 1-p_{j}-f_{j}^{\max } ; j=1,2, \ldots, m \\
\mathbf{k}_{j}^{T} \boldsymbol{\alpha} & \leq 1-f_{j}^{\max } ; \quad j=1,2, \ldots, m \\
\mathbf{c}^{T} \boldsymbol{\alpha} & \geq 0 \\
\mathbf{c}^{T} \boldsymbol{\alpha} & \leq 1 \\
\alpha_{0} & \geq 0 \\
\alpha_{0} & \leq 1 \\
\mathbf{\Phi}_{\mathbf{k}}{ }^{T} \boldsymbol{\alpha} & \geq 0 \quad ; \quad k=0,1, \ldots, N_{p}-1
\end{aligned}
$$

com as quantidades $\mathbf{d}, \mathbf{k}_{j}, \mathbf{c}, \boldsymbol{\Phi}_{\mathbf{k}}, f_{j}^{\max }$ e $\boldsymbol{\alpha}$ já definidas anteriormente nesta seção.

Note que trata-se de um problema de otimização linear com restrições lineares. O espaço de soluções viáveis é a interseção de regiões limitadas por hiperplanos, sendo, portanto, convexo. Além disso, a função objetivo é linear, sendo suas superfícies de nível hiperplanos perpendiculares ao vetor $\mathbf{d}$. A solução obtida corresponde então ao máximo global e está localizada na fronteira do espaço de soluções viáveis.

\section{Resultados NumÉRICOS}

De modo a ilustrar a aplicação do procedimento desenvolvido na obtenção de máscaras a serem satisfeitas pelo comportamento estatístico das interferências externas, é considerada uma situação específica envolvendo um enlace por satélite operando em $19 \mathrm{GHz}$. Exemplos de aplicação que consideram enlaces em outras faixas de frequiência e enlaces que utilizam controle automático de potência (APC) podem ser encontrados em [2].

A base de funções ortonormais $\left\{\phi_{i}(Y), i=1, \ldots, n\right\}$ utilizada para a representação de $p_{y}(Y)$ é dada por

$\phi_{i}(Y)=\frac{1}{K} \bar{P}_{i-1}\left(\frac{Y-Y_{\min }}{Y_{\max }-Y_{\min }}\right) ; \quad \begin{aligned} & Y_{\min }<Y<Y_{\max } \\ & i=1,2, \ldots, n\end{aligned}$

onde $K=\sqrt{Y_{\max }-Y_{\min }}$ e $\left\{\bar{P}_{i} ; i=1,2, \ldots, n\right\}$ são Polinômios de Legendre Deslocados (shifted Legendre Polynomials) [3], normalizados para energia unitária. No exemplo apresentado considerou-se $Y_{\min }=0, Y_{\max }=5$ e $n=7$ (valores maiores de $n$ conduziram a resultados bem próximos do correspondente a $n=7$ ).

A distribuição cumulativa de probabilidade da degradação devido a chuvas foi obtida considerando-se o modelo de atenuação por chuvas estabelecido na Recomendação ITU-R P.618-7 [4]. Foi utilizado o procedimento descrito no Item 2.2.1.1 dessa Recomendação, no qual foram considerados os parâmetros apresentados na Tabela I. A distribuição cumulativa de probabilidade obtida é aquela já mostrada na Figura 1. Os requisitos de desempenho associados aos valores da razão

TABELA I

ParÂMETros CONSIDERAdos NO ENLACE EM 19 GHz

\begin{tabular}{|l|r|}
\hline taxa de precipitação pluviométrica $-R_{0.01}-[\mathrm{mm} / \mathrm{h}]$ & 23 \\
\hline altitude acima do nível do mar da estação terrena receptora $-h_{s}-[\mathrm{km}]$ & 0 \\
\hline altura da chuva $-h_{r}-[\mathrm{km}]$ & 3 \\
\hline ângulo de elevação da antena da estação terrena receptora $-\theta-$ [graus] & 25 \\
\hline latitude da estação terrena receptora $-\varphi-[$ graus] & 40 \\
\hline freqüência de operação do enlace $-f-[\mathrm{GHz}]$ & 19 \\
\hline raio efetivo da Terra $-R_{e}-[\mathrm{km}]$ & 8500 \\
\hline
\end{tabular}

$E_{b} / N_{0}$, expressos em (5), são mostrados na Tabela II. A razão

TABELA II

REQUiSITOS DE DESEMPENHO PARA O ENLACE DE $19 \mathrm{GHZ}$

\begin{tabular}{|c|c|c|}
\hline$B E R_{j}$ & $\left(E_{b} / N_{0}\right)_{j}(\mathrm{~dB})$ & $p_{j}$ \\
\hline \hline $1 \times 10^{-6}$ & 6,5 & 0,0004 \\
\hline $1 \times 10^{-8}$ & 7,6 & 0,006 \\
\hline $1 \times 10^{-9}$ & 8,7 & 0,04 \\
\hline
\end{tabular}

$E_{b} / N_{0}$ em céu claro foi determinada considerando-se (12) e (10), ou seja, determinou-se o menor valor de $\left(E_{b} / N_{0}\right)_{C S}$ que satisfaz às condições

$$
1-F_{x}\left(\left(E_{b} / N_{0}\right)_{C S}-\left(E_{b} / N_{0}\right)_{j}\right) \leq 0,9 p_{j} \quad ; j=1, \ldots, 3
$$

A restrição em (48) foi considerada utilizando-se 101 pontos no intervalo $\left[Y_{\min }, Y_{\min }\right]$, ou seja, $N_{p}=101$. A função objetivo utilizada, definida em (53), considerou a possibilidade de ocorrência de valores de degradação $y$ em todo o intervalo, ou seja, $\mathcal{S}=\left(Y_{\min }, Y_{\max }\right)$.

Partindo-se da condição inicial em que a degradação devido a interferências externa é zero com probabilidade 1, ou seja, $\boldsymbol{\alpha}_{0}=\left(\begin{array}{llll}1 & 0 & \cdots & 0\end{array}\right)^{T}$, obteve-se, como solução do problema de otimização definido em (53) e (54), o vetor $\boldsymbol{\alpha}^{*}$, dado por

$$
\begin{array}{r}
\boldsymbol{\alpha}^{*}=\left(\begin{array}{cccccc}
0,0909 & 0,4070 & -0,6040 & 0,5782 & -0,4304 \\
0,2509 & -0,1058 & 0,0257 & 0,0000
\end{array}\right)^{T}
\end{array}
$$

A função densidade de probabilidade $p_{y}(Y)$ correspondente a esta solução é apresentada na Figura 2. Note que na solução encontrada a chance de ocorrência de degradação devido a interferências no intervalo $\left(Y_{\min }, Y_{\max }\right]$ é de, aproximadamente, $90 \%$. A distribuição cumulativa de probabilidade da degradação $y$ devido a interferências foi obtida integrandose $p_{y}((Y)$ e é apresentada na Figura 3. Com base no relacionamento indicado em (20), entre a função distribuição de probabilidade da razão $i / n$ e a função distribuição de probabilidade da degradação $y$ devido a interferências externas, foi obtida a distribuição cumulativa de probabilidade da razão $i / n$, mostrada na Figura 4. Note que a distribuição cumulativa de probabilidade da Figura 3 pode ser vista como uma máscara que limita o comportamento estatístico da degradação devido a interferências externas. Isto porque os requisitos de 


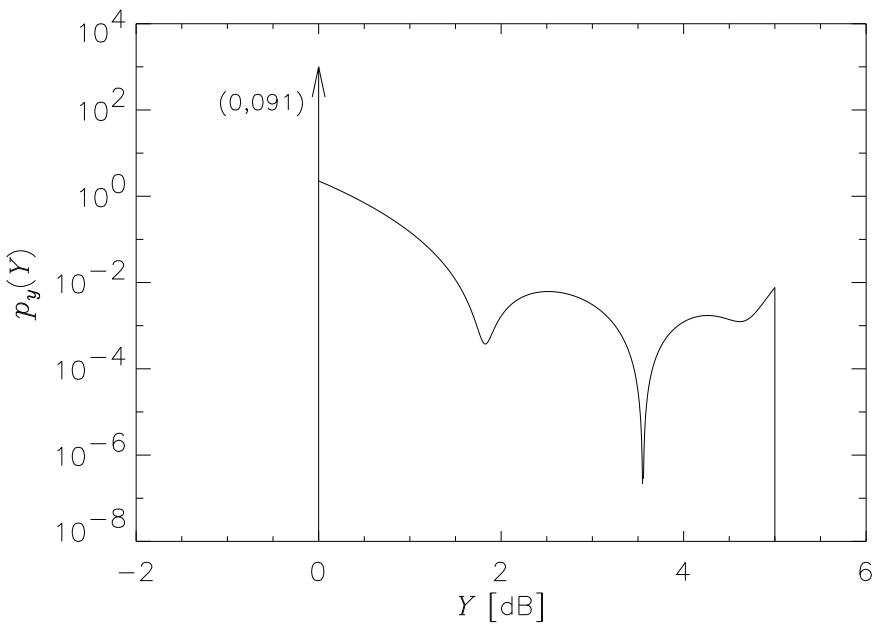

Fig. 2. Função densidade de probabilidade da degradação devido a interferências externas para o enlace de $19 \mathrm{GHz}$.

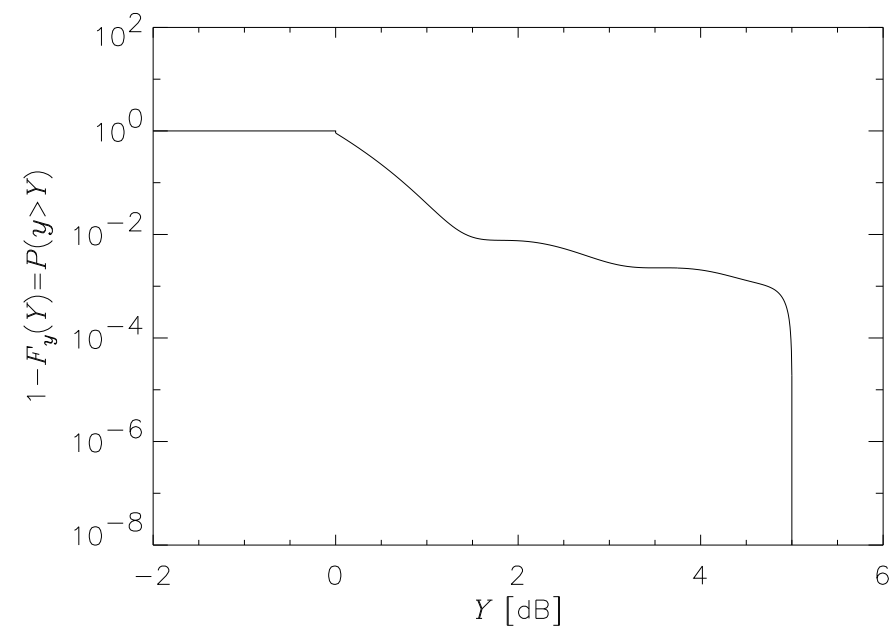

Fig. 3. Distribuição cumulativa de probabilidade da degradação devido a interferências externas para o enlace de $19 \mathrm{GHz}$.

desempenho em (1) são satisfeitos para qualquer degradação cujo comportamento estatístico corresponda a uma distribuição cumulativa de probabilidade que esteja abaixo da curva da figura 3. Este comentário aplica-se também à curva da figura 4, que pode ser também vista como uma máscara que limita o comportamento estatístico da razão interferência-ruído térmico $i / N$.

\section{Conclusĩ̃o}

Foi desenvolvida uma técnica capaz de determinar, de maneira sistemática, as limitações (máscaras) a serem impostas ao comportamento estatístico do agregado de interferências que afeta um enlace via satélite de modo a garantir um desempenho adequado ao enlace. A técnica proposta considera, como as metodologias apresentadas em [1], o efeito conjunto das degradações devido a chuvas e a interferências externas. A caracterização da função densidade de probabilidade da degradação devido a interferências é feita através de uma representação em base de funções ortonormais e a máscara que

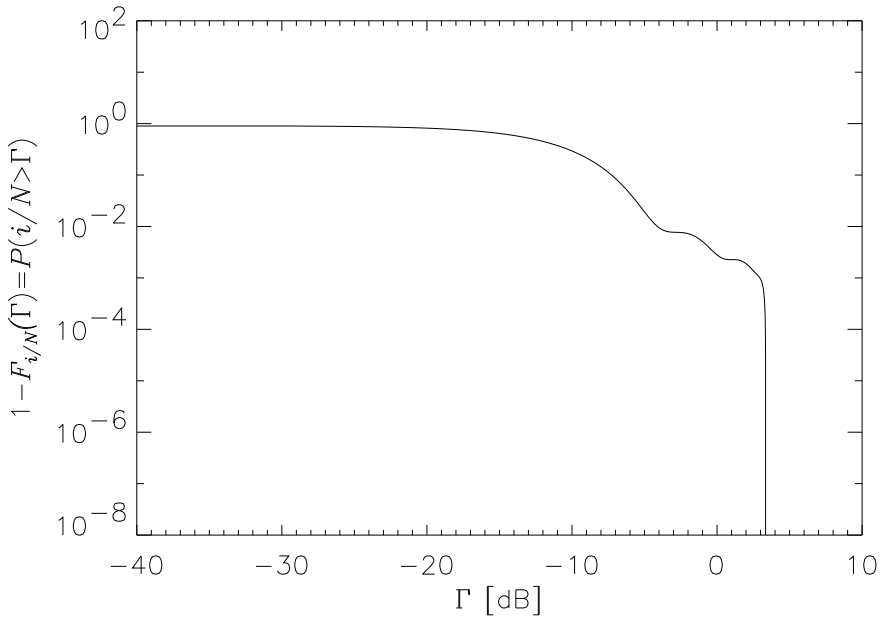

Fig. 4. Distribuição cumulativa de probabilidade da razão $i / n$ para o enlace de $19 \mathrm{GHz}$ (possível máscara para o comportamento estatístico da razão $i / n$ ).

limita o comportamento estatístico da interferência agregada é obtida através da solução de um problema de otimização com restrições conveniente, definido na Seção III. Além disso, a técnica proposta permite a utilização dos modelos usualmente empregados na estimação da atenuação por efeito de chuvas e a consideração de funções densidade de probabilidade bastante genéricas para a degradação devido a interferências externas.

Foi apresentado um exemplo de aplicação da técnica proposta a uma situação bastante realista envolvendo um enlace via satélite na faixa de $19 \mathrm{GHz}$. Neste exemplo específico, partindo-se de uma condição inicial que considerava que a degradação devido a interferencias, expressa em $\mathrm{dB}$, era zero probabilidade 1 , ou seja, não há interferências, a técnica proposta conduziu a uma máscara que permite que a degradação devido a interferências tome valores entre 0 e $5 \mathrm{~dB}$ com probabilidade significativa $(P(y \in(0,5))>0,9)$. A partir deste resultado foi obtida uma máscara para a limitação do comportamento estatístico da razão interferência-ruído térmico.

Exemplos de aplicação que consideram enlaces em outras faixas de freqüência e enlaces que utilizam controle automático de potência (APC) podem ser encontrados em [2].

\section{REFERÊNCIAS}

[1] Maximum permissible levels of interference in a satellite network (GSO/FSS; non-GSO/FSS; non-GSO/MSS feeder links) in the fixedsatellite service caused by other codirectional FSS networks below $30 \mathrm{GHz}$, Recomendação ITU-R S.1323-2, União Internacional de Telecomunicações, Genebra, 2002.

[2] Baptista, A. J. S., Desempenho de enlaces de satélites em presença de chuvas e interferências externas: restrições a serem impostas ao comportamento estatístico das interferências, Dissertação de Mestrado, DEE, PUC-Rio, agosto 2007.

[3] Abramowitz M., Stegun I. A., Handbook of Mathematical Functions, Dover Publication Inc., New York, 9th Edition, 1972.

[4] Propagation data and prediction methods required for the design of Earth-space telecommunication systems, Recomendação ITU-R P.6187, União Internacional de Telecomunicações, Genebra, 2001. 